\title{
Correction to: An Evaluation of the Firefighting Effectiveness of Fluorine-Free Foams
}

Gerard G. Back*, Jensen Hughes, Inc., 3610 Commerce Drive, Suite 817, Baltimore, MD 21227, USA

Published online: 13 February 2021

\section{Correction to: Fire Technology (c) 2020 Springer Science + Business Media, LL, part of Springer Nature Manufactured in The United States https://doi.org/10.1 007/s 10694-020-01051-4}

In the original publication of the article, Table 1, 2, 3 was incorrectly published. The corrected version is given belowe. The original article has been corrected.

The revised Tables 1, 2 and 3 are as follows.

Table 1

Foam Quality/Aspiration Comparison

\begin{tabular}{|c|c|c|c|c|c|c|c|}
\hline \multirow[b]{2}{*}{ Foam } & \multirow[b]{2}{*}{$\begin{array}{l}\text { Test } \\
\text { Type }\end{array}$} & \multirow[b]{2}{*}{$\begin{array}{l}\text { Fuel } \\
\text { Type }\end{array}$} & \multirow[b]{2}{*}{$\begin{array}{l}\text { Water } \\
\text { Type }\end{array}$} & \multicolumn{2}{|c|}{$\begin{array}{c}\text { Lower Aspiration } \\
\text { (3-4 exp) }\end{array}$} & \multicolumn{2}{|c|}{$\begin{array}{c}\text { Higher Aspiration } \\
\text { (7-8 exp) }\end{array}$} \\
\hline & & & & \begin{tabular}{|c|} 
App. \\
Rate \\
$\mathrm{gpm} / \mathrm{ft}^{2}$
\end{tabular} & $\begin{array}{c}\text { Ext } \\
\text { Density } \\
\text { gal/ft }^{2}\end{array}$ & $\begin{array}{c}\text { App. } \\
\text { Rate } \\
\mathrm{gpm} / \mathrm{ft}^{2}\end{array}$ & $\begin{array}{c}\text { Ext } \\
\text { Density } \\
\text { gal/ft }{ }^{2}\end{array}$ \\
\hline AR-FFF1 & Type III & MIL-SPEC & Fresh & 0.09 & 0.450 & 0.06 & 0.250 \\
\hline AR-FFF1 & Type III & MIL-SPEC & Salt & 0.09 & 0.375 & 0.06 & 0.260 \\
\hline AR-FFF2 & Type III & MIL-SPEC & Fresh & 0.09 & 0.390 & 0.06 & 0.255 \\
\hline AR-FFF2 & Type III & MIL-SPEC & Salt & 0.09 & 0.375 & 0.06 & 0.270 \\
\hline H-FFF1 & Type III & MIL-SPEC & Fresh & 0.06 & 0.165 & 0.06 & 0.150 \\
\hline H-FFF1 & Type III & MIL-SPEC & Salt & 0.06 & 0.290 & 0.06 & 0.210 \\
\hline \begin{tabular}{|l|}
$\mathrm{H}-\mathrm{FFF} 2$ \\
\end{tabular} & Type III & MIL-SPEC & Fresh & 0.06 & 0.187 & 0.06 & 0.160 \\
\hline
\end{tabular}

The original article can be found online at https://doi.org/10.1007/s10694-020-01051-4.

\footnotetext{
*Correspondence should be addressed to: Gerard G. Back, E-mail: jback@haifire.com
} 
Table 2

Fuel Type Comparison (Higher Aspirated Foam/Freshwater)

\begin{tabular}{|c|c|c|c|c|c|c|c|c|}
\hline \multirow[b]{2}{*}{ Foam } & \multicolumn{2}{|c|}{ Heptane } & \multicolumn{2}{|c|}{ MIL-SPEC } & \multicolumn{2}{|c|}{ E10 } & \multicolumn{2}{|c|}{ IPA } \\
\hline & $\begin{array}{c}\text { App. } \\
\text { Rate } \\
\text { gpm/ft' }\end{array}$ & $\begin{array}{c}\text { Ext. } \\
\text { Density } \\
\text { gal/ft }\end{array}$ & $\begin{array}{c}\text { App. } \\
\text { Rate } \\
\text { gpm/ft² }\end{array}$ & $\begin{array}{c}\text { Ext. } \\
\text { Density } \\
\text { gal/ft }\end{array}$ & $\begin{array}{l}\text { App. } \\
\text { Rate } \\
\text { gpm/ft² }\end{array}$ & $\begin{array}{c}\text { Ext. } \\
\text { Density } \\
\text { gall/ft }\end{array}$ & $\begin{array}{l}\text { App. } \\
\text { Rate } \\
\text { gpm/ft }\end{array}$ & $\begin{array}{c}\text { Ext. } \\
\text { Density } \\
\text { gal/ft }\end{array}$ \\
\hline AR-AFFF & 0.04 & 0.051 & 0.06 & 0.065 & 0.06 & 0.060 & 0.045 & 0.203 \\
\hline \begin{tabular}{|l|} 
AR-FFF1 \\
\end{tabular} & 0.06 & 0.105 & 0.06 & 0.220 & 0.09 & 0.413 & 0.16 & 0.733 \\
\hline \begin{tabular}{|l|} 
AR-FFF2 \\
\end{tabular} & 0.06 & 0.107 & 0.06 & 0.210 & 0.09 & 0.383 & 0.16 & 0.787 \\
\hline \begin{tabular}{|l|} 
AR-FFF3 \\
\end{tabular} & 0.06 & NT & 0.06 & 0.230 & 0.09 & 0.353 & 0.10 & 0.450 \\
\hline \begin{tabular}{|l|}
$\mathrm{H}-\mathrm{FFF} 1$ \\
\end{tabular} & 0.06 & 0.092 & 0.06 & 0.185 & 0.075 & 0.356 & NT & NT \\
\hline \begin{tabular}{|l|}
$\mathrm{H}$-FFF2 \\
\end{tabular} & 0.06 & 0.055 & 0.06 & 0.170 & 0.075 & 0.363 & NT & NT \\
\hline
\end{tabular}

\section{Table 3}

Fuel Temperature and Water Type Comparison (Type III, MIL-SPEC, Higher Aspirated Foam)

\begin{tabular}{|c|c|c|c|c|c|c|}
\hline \multirow[b]{2}{*}{ Foam } & \multirow[b]{2}{*}{ Water Type } & \multirow[b]{2}{*}{ Flow Rate } & \multicolumn{2}{|c|}{$48^{\circ}-66^{\circ} \mathrm{F}$} & \multicolumn{2}{|c|}{$82^{\circ}-93^{\circ} \mathrm{F}$} \\
\hline & & & $\begin{array}{l}\text { Cont } \\
\text { Time }\end{array}$ & $\begin{array}{c}\text { Ext } \\
\text { Time }\end{array}$ & $\begin{array}{l}\text { Cont } \\
\text { Time }\end{array}$ & $\begin{array}{l}\text { Ext } \\
\text { Time }\end{array}$ \\
\hline AR-FFF1 & Fresh & $3.75 \mathrm{gpm}$ & $0: 50$ & $3: 30$ & 1:00 & $2: 50$ \\
\hline AR-FFF1 & Salt & $3.75 \mathrm{gpm}$ & 1:00 & $3: 15$ & 1:00 & $2: 55$ \\
\hline AR-FFF2 & Fresh & $3.75 \mathrm{gpm}$ & $1: 15$ & $3: 45$ & 1:00 & $3: 45$ \\
\hline AR-FFF2 & Salt & $3.75 \mathrm{gpm}$ & $1: 10$ & $3: 40$ & $1: 05$ & $3: 25$ \\
\hline AR-FFF3 & Fresh & $3.75 \mathrm{gpm}$ & $0: 45$ & $3: 20$ & $1: 05$ & $3: 40$ \\
\hline FFF1 & Fresh & $3.0 \mathrm{gpm}$ & $0: 45$ & $2: 30$ & 1:00 & $3: 05$ \\
\hline FFF1 & Salt & $3.0 \mathrm{gpm}$ & $1: 05$ & $3: 30$ & 1:00 & $2: 55$ \\
\hline FFF2 & Fresh & $3.0 \mathrm{gpm}$ & $0: 45$ & $2: 40$ & $1: 10$ & $2: 50$ \\
\hline
\end{tabular}

